\title{
Discharging/Charging Voltage-Temperature Pattern Recognition for Improved SOC/Capacity Estimation and SOH Prediction at Various Temperatures
}

\author{
Jonghoon $\mathrm{Kim}^{\dagger}$, Seongjun Lee*, and Bohyung Cho** \\ $\dagger^{* *}$ Dept. of Electrical Eng., Seoul National University, Seoul, Korea \\ ${ }^{*}$ R\&D Center, Samsung Techwin, Seongnam, Korea
}

\begin{abstract}
This study investigates an application of the Hamming network-dual extended Kalman filter (DEKF) based on pattern recognition for high accuracy state-of-charge (SOC)/capacity estimation and state-of-health $(\mathrm{SOH})$ prediction at various temperatures. The averaged nine discharging/charging voltage-temperature (DCVT) patterns for ten fresh Li-Ion cells at experimental temperatures are measured as representative patterns, together with cell model parameters. Through statistical analysis, the Hamming network is applied to identify the representative pattern that matches most closely with the pattern of an arbitrary cell measured at any temperature. Based on temperature-checking process, model parameters for a representative DCVT pattern can then be applied to estimate SOC/capacity and to predict SOH of an arbitrary cell using the DEKF. This avoids the need for repeated parameter measuremet.
\end{abstract}

Key Words: Dual extended Kalman filter (DEKF), Hamming network, Pattern recognition, State-of-charge (SOC), State-of-health $(\mathrm{SOH})$

\section{INTRODUCTION}

A battery management system (BMS) is critical for maintaining cell performance [1], [2]. Specifically, in electric vehicles (EV) and hybrid electric vehicles (HEV) applications, it is important to have information about the cell's state-ofcharge (SOC) [3], [4], capacity [5], [6], and state-of-health $(\mathrm{SOH})$ based on the pulse power analysis [7], [8]. Precise SOC, capacity, and SOH information are critical in practical applications where it is necessary to determine how long the cell will last and, importantly, when to stop charging and discharging, as over-charging and over-discharging may cause permanent internal damage [9].

In recent years, much research has been devoted to developing improved methods for SOC/capacity estimation and SOH prediction. Specifically, the dual extended Kalman filter (DEKF), which makes use of two extended Kalman filters (EKFs) running in parallel, is widely used for state and parameter estimations [10]. The accuracy of the DEKF depends largely on the predetermined parameter values used in the equivalent circuit model shown in Fig. 1. It is important to accurately measure model parameters that include open circuit voltage $(\mathrm{OCV})$, series resistance $\left(\mathrm{R}_{i}\right)$, diffusion

\footnotetext{
Manuscript received Jul. 31, 2011; revised Oct. 3, 2011

Recommended for publication by Associate Editor Woo-Jin Choi.

$\dagger$ Corresponding Author: qwzxas@ @anmail.net

Tel: +82-2-880-1785, Fax: +82-2-878-1452, Seoul Nat'l University

* R\&D Center, Samsung Techwin, Korea

** Dept. of Electrical Eng., Seoul National University, Korea
}

resistance $\left(\mathrm{R}_{\text {Diff }}\right)$, and diffusion capacitance $\left(\mathrm{C}_{\text {Diff }}\right)$ [11], [12]. Because these parameters vary with temperature, the accuracy of SOC/capacity estimation and $\mathrm{SOH}$ prediction method will also vary. The error can be reduced by repeating parameter measurement, but such an exercise would be very time- consuming and inefficient. Thus, the existing DEKF algorithm can only be applied to a single cell under controlled experimental conditions [11], [12]. It should be considered that the model parameters could be changed for correct SOC/ capacity estimation and $\mathrm{SOH}$ prediction.

This study proposes an application of the DEKF that performs high accuracy SOC/capacity estimation and $\mathrm{SOH}$ prediction based on pattern recognition at various temperatures. In this study, the Hamming network [13], [14] generally used for binary pattern recognition is utilized to evaluate several predetermined representative discharging/charging voltage- temperature (DCVT) patterns, and determine which is the closest to the input DCVT pattern by comparing the inner product. Representative DCVT patterns are collected from nine average discharging/charging voltages from ten Li-Ion cells. Based on the temperature-checking process, the model parameters determined from the representative DCVT pattern are then applied to estimate SOC/capacity and predict $\mathrm{SOH}$ of an arbitrary cell using the DEKF. This avoids the need for repeated parameter measurement. Experimental studies were conducted on Samsung 18650 Li-Ion cells with a total capacity of $1.3 \mathrm{Ah}$. 


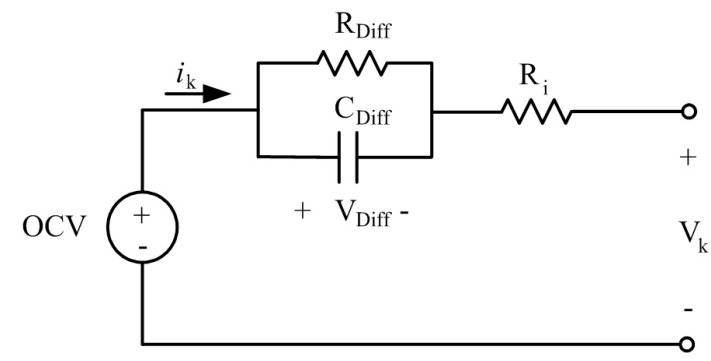

Fig. 1. Lumped parameter cell model: open circuit voltage (OCV), series resistance $\left(\mathrm{R}_{i}\right)$, diffusion resistance $\left(\mathrm{R}_{\text {Diff }}\right)$, and diffusion capacitance $\left(\mathrm{C}_{\text {Diff }}\right)$.

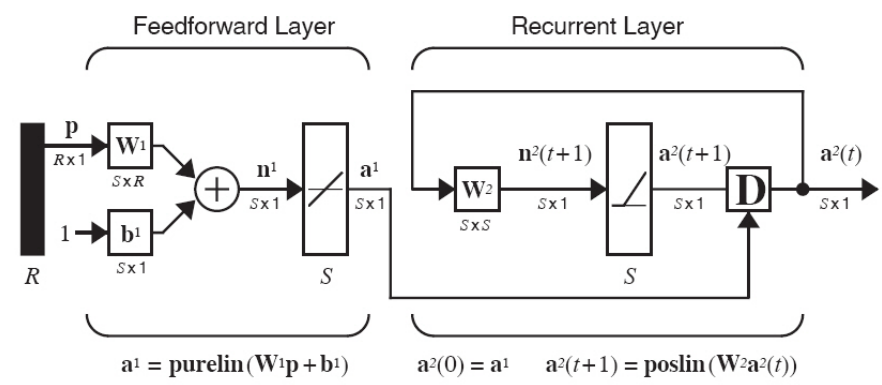

Fig. 2. Hamming network.

\section{HAMMING NeTWORK}

The Hamming network [13], [14] is used for pattern recognition, as shown in Fig. 2. It is one of the simplest competitive networks and is designed explicitly to solve binary pattern recognition issues. The Hamming network decides which representative pattern is closest to the current pattern by comparing the inner products. Its objective is to decide which prototype vector is closest to the input vector. The Hamming network consists of two layers: the feedforward layer and the recurrent layer.

\section{A. Feedforward Layer}

The feedforward layer performs a correlation or inner product between each prototype pattern and a current pattern in order to search a minimum Hamming distance (HD) from calculation the difference between dimension $m$ and HD. In order to perform the inner product, weight matrix $\mathbf{W}^{1}$ is set to prototype vectors and is transformed into the binary form, in addition to bias vector, and is transformed into the binary form, in addition to bias vector, $\mathbf{b}^{1}$, in (1) and (2).

$$
\begin{gathered}
\mathbf{W}^{1}=\left[\begin{array}{c}
1 \mathbf{w}^{i T} \\
2 \mathbf{w}^{i T} \\
\vdots \\
{ }_{S} \mathbf{w}^{i T}
\end{array}\right]=\frac{1}{2}\left[\begin{array}{cccc}
1 \mathbf{w}^{1} & { }_{2} \mathbf{w}^{1} & \cdots & { }_{S} \mathbf{w}^{1} \\
1 \mathbf{w}^{2} & { }_{2} \mathbf{w}^{2} & \cdots & { }_{S} \mathbf{w}^{2} \\
\vdots & \vdots & \ddots & \vdots \\
1 \mathbf{w}^{R} & { }_{2} \mathbf{w}^{R} & \cdots & { }_{S} \mathbf{w}^{R}
\end{array}\right]=\left[\begin{array}{c}
\mathbf{p}_{l}^{T} \\
\mathbf{p}_{2}^{T} \\
\vdots \\
\mathbf{p}_{S}^{T}
\end{array}\right] \\
\mathbf{b}^{1}=[R, R, \cdots, R]^{T}=\left[\frac{m}{2}, \frac{m}{2}, \cdots, \frac{m}{2}\right]^{T}
\end{gathered}
$$

where each row of $\mathbf{W}^{1}$ represents a prototype vector which it is required to be recognize, and each element of $\mathbf{b}^{1}, m / 2$ is the threshold value and is set equal to the number of elements in each input vector $R, S$ is the number of neurons. As expressed in (3), it is high desirable to have the $i^{\text {th }}(1 \leq i \leq R)$ node in this layer compute $m-H D\left({ }_{i} \mathbf{w}, \mathbf{p}\right)$ for a given input vector $\mathbf{p}$, where $H D\left({ }_{i} \mathbf{w}, \mathbf{p}\right)$ is the Hamming distance between vectors ${ }_{i} \mathbf{w}$ and $\mathbf{p}$. Then, the net input of node is as in (4), namely, the feedforward layer output. After all, these outputs are equal to the inner products of the prototype vectors with the input, plus $R$. In the feedforward layer, a linear transfer function (purelin) [14] is used to implement these inner products in (5). The neuron in this layer with the largest output corresponds to the prototype pattern that is closest in Hamming distance to the input pattern.

$$
\begin{gathered}
\mathbf{W}^{1} \mathbf{p}=\left[m-H D\left({ }_{i} \mathbf{w}, \mathbf{p}\right)\right]-H D\left({ }_{i} \mathbf{w}, \mathbf{p}\right) \\
\text { net }_{i}=\mathbf{n}^{1}=\mathbf{W}^{\mathbf{1}} \mathbf{p}+\frac{m}{2}=m-H D\left({ }_{i} \mathbf{w}, \mathbf{p}\right) \quad \mathbf{i}=1,2, \cdots, S \\
\mathbf{a}^{1}=\mathbf{W}^{1} \mathbf{p}+\mathbf{b}^{1}=\left[\begin{array}{c}
\mathbf{p}_{l}^{T} p+R \\
\mathbf{p}_{2}^{T} p+R \\
\vdots \\
\mathbf{p}_{S}^{T} p+R
\end{array}\right]=\operatorname{purelin}\left(\mathrm{W}^{1} \mathbf{p}+\mathbf{b}^{1}\right) .
\end{gathered}
$$

\section{B. Recurrent Layer}

The recurrent layer is known as the MAXNET [14] and a competitive layer which performs the winner-take-all (WTA) operation, whose purpose is to enhance the initial dominant response of the $i^{\text {th }}$ node and suppress the others [14]. As expressed in (6), the neurons and initialized with the outputs of the feedforward layer, which indicate the correlation between the prototype vectors and the input vector.

$$
\mathbf{a}^{2}(0)=\mathbf{a}^{1}
$$

As a result of recurrent processing, the $i^{\text {th }}$ node responds positively while the responses of all remaining nodes decay to zero. Namely, in order to determine a winner, which only has a positive output, the neurons compete with each other. Then, as expressed in (7), the recurrent layer output is updated according to the following recurrence relation which uses a positive transfer function [14].

$$
\mathbf{a}^{2}(t+1)=\operatorname{poslin}\left(W^{2} \mathbf{a}^{2}(t)\right) .
$$

This processing requires self-feedback connections and negative lateral inhibition connections, in which the output of each neuron has an inhibitory effect on all of the other neurons [14]. The $n \times n$ weight matrix of the recurrent layer $\mathbf{W}^{2}$ is taken in (8). The weights in this layer are set so that the diagonal elements are 1, and the off-diagonal elements have a small negative value, where $0<\varepsilon<1 /(S-1)$ is called the lateral interaction coefficient. Thus, it can be substituted weight values of 1 and $-\varepsilon$ for the appropriate elements of $\mathbf{W}^{2}$ in (9), where $1 \leq i \leq S$ and $1 \leq j \leq S$.

$$
\begin{gathered}
\mathbf{W}^{2}=\left[\begin{array}{cccc}
{ }_{1} \mathbf{w}^{1} & { }_{1} \mathbf{w}^{2} & \cdots & { }_{1} \mathbf{w}^{S} \\
{ }_{2} \mathbf{w}^{1} & { }_{2} \mathbf{w}^{2} & \cdots & { }_{2} \mathbf{w}^{S} \\
\vdots & \vdots & \ddots & \vdots \\
{ }_{i} \mathbf{w}^{1} & { }_{i} \mathbf{w}^{2} & \cdots & { }_{i} \mathbf{w}^{S}
\end{array}\right]=\left[\begin{array}{cccc}
1 & -\varepsilon & \cdots & -\varepsilon \\
-\varepsilon & 1 & \cdots & -\varepsilon \\
\vdots & \vdots & \ddots & \vdots \\
-\varepsilon & -\varepsilon & \cdots & 1
\end{array}\right. \\
\mathbf{a}_{i}^{2}(\mathrm{t}+1)=\operatorname{poslin}\left(\mathbf{a}_{\mathrm{i}}^{2}(\mathrm{t})-\varepsilon \sum_{\mathrm{j} \neq \mathrm{i}} \mathbf{a}_{\mathrm{j}}^{2}(\mathrm{t})\right)
\end{gathered}
$$




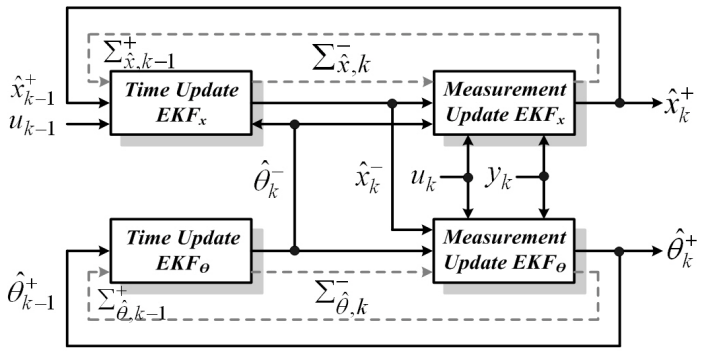

Fig. 3. Dual extended Kalman filter (DEKF).

Each neuron's output decreases in proportion to the sum of the other neuron's outputs. The output of the neuron with the largest initial condition decreases more slowly than the outputs of the other neurons. Eventually, only one neuron has a positive output. The index of the recurrent layer neuron with a stable positive output is the index of the prototype vector that best matched the input.

\section{Dual Extended Kalman Filter (DEKF)}

The DEKF is used to estimate the SOC/capacity of a Li-Ion cell. This algorithm combines the two EKFs, one of which is the state filter, which estimates the SOC, and the other is the weight filter, which estimates the capacity. At every time step, the state filter uses a priori value of the weight filter, while the weight filter uses a priori value of the state filter. Therefore, the two EKFs are calculated concurrently to estimate SOC/ capacity. The DEKF can be reviewed by drawing a block diagram, as shown in Fig. 3.

The state-space representation with difference equations of the DEKF is described in (10)-(12).

$$
\begin{gathered}
x_{k+1}=f_{x}\left(x_{k}, u_{k}, \theta_{k}\right)+w_{k}^{x} \quad w_{k}^{x} \sim N\left(0, Q_{k}^{x}\right) \\
\theta_{k+1}=\theta_{k}+w_{k}^{\theta} \quad w_{k}^{\theta} \sim N\left(0, Q_{k}^{\theta}\right) \\
y_{k+1}=h_{k}\left(x_{k}, u_{k}, \theta_{k}\right)+v_{k} \quad v_{k} \sim N\left(0, R_{k}\right) .
\end{gathered}
$$

The symbols $w_{k}^{x}$ and $w_{k}^{\theta}$ represent the process noise of the state filter and weight filter, respectively, are measured to be independent, zero-mean, Gaussian noise with covariance matrices $Q_{k}^{x}$ and $Q_{k}^{\theta}$. The measurement noise $v_{k}$ is assumed to be independent, zero-mean, Gaussian noise with a covariance matrix $R_{k}$. Then the equations that decide the Kalman gain $K_{k}^{x}$ and $K_{k}^{\theta}$ of the state filter and weight filter are as follows:

$$
\begin{aligned}
K_{k}^{x} & =P_{\hat{x}_{k}}^{-}\left(H_{k}^{x}\right)^{\mathrm{T}}\left[H_{k}^{x} P_{\hat{x}_{k}}^{-}\left(H_{k}^{x}\right)^{\mathrm{T}}+R_{k}\right]^{-1} \\
K_{k}^{\theta} & =P_{\hat{\theta}_{k}}^{-}\left(H_{k}^{\theta}\right)^{\mathrm{T}}\left[H_{k}^{\theta} P_{\hat{\theta}_{k}}^{-}\left(H_{k}^{\theta}\right)^{\mathrm{T}}+R_{k}\right]^{-1}
\end{aligned}
$$

where the error covariance is $P_{k}$ and the measurement sensitivity matrix is $H_{k}$.

The state-space equation of the cell model is derived as Eqs. (15)-(17), from the equivalent circuit shown in Fig. 1 and $\Delta t$ is time between step $k$ and $k+1$.

$$
\begin{aligned}
x_{k+1} & =\left[\begin{array}{c}
\mathrm{SOC}_{k+1} \\
V_{\text {Diff }, k+1}
\end{array}\right]=\left[\begin{array}{cc}
1 & 0 \\
0 & 1-\frac{\Delta t}{R_{\text {Diff }} C_{\text {Diff }}}
\end{array}\right]\left[\begin{array}{c}
S O C_{k} \\
V_{\text {Diff }, k}
\end{array}\right] \\
& +\left[\begin{array}{c}
-\frac{\Delta t}{C_{n, k}} \\
\frac{\Delta t}{C_{\text {Diff }}}
\end{array}\right] i_{k}+w_{k}^{x}
\end{aligned}
$$

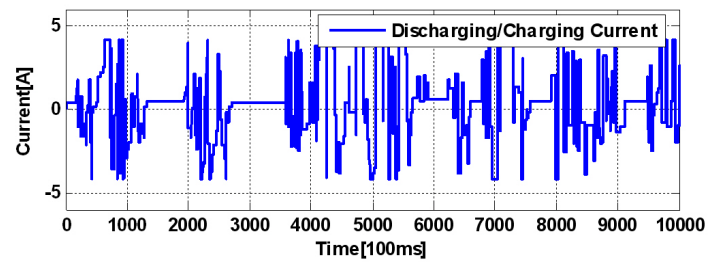

Fig. 4. Current profile for obtaining the DCVT pattern.

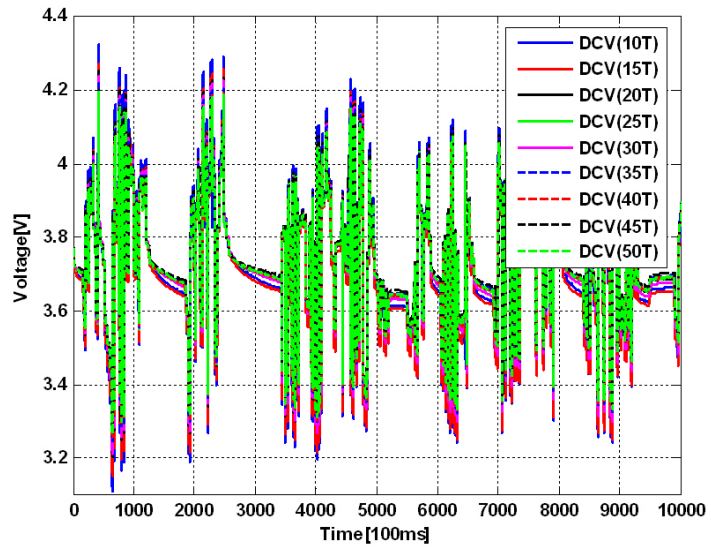

Fig. 5. Average DCVT patterns for ten fresh Li-Ion cells at various temperatures: $10-50^{\circ} \mathrm{C}$ with a $5^{\circ} \mathrm{C}$ temperature interval.

$$
\begin{gathered}
\theta_{k}=\left[\begin{array}{l}
\mathrm{C}_{\mathrm{n}, k+1} \\
\mathrm{R}_{\mathrm{i}, k+1}
\end{array}\right]=\left[\begin{array}{ll}
1 & 0 \\
0 & 1
\end{array}\right]\left[\begin{array}{l}
\mathrm{C}_{\mathrm{n}, k} \\
\mathrm{R}_{\mathrm{i}, k}
\end{array}\right]+w_{k}^{\theta} \\
V_{k}=\operatorname{OCV}\left(\operatorname{SOC}_{k}, C_{n, k}\right)-V_{\text {Diff }, k}-R_{i} i_{k} .
\end{gathered}
$$

The OCV is the measurement equation is implemented by the relationship of the OCV-SOC data. The measurement matrix derived from Eqs. (18)-(21). From Eq. (20), the measurement matrix of the capacity $\mathrm{C}_{n}$ requires the total differential because the OCV is a function of the SOC. In this case, the first term of the right-hand side of Eq. (20) is irrelevant to the capacity. The measurement matrix of the series resistance $\mathrm{R}_{i}$ can be obtained from Eq. (21).

$$
\begin{gathered}
H_{k}^{\mathrm{SOC}}=\frac{\partial V_{t}}{\partial \mathrm{SOC}}=\frac{\partial \mathrm{OCV}}{\partial \mathrm{SOC}} \\
H_{k}^{\mathrm{V}_{\text {Diff }}}=\frac{\partial V_{t}}{\partial \mathrm{V}_{\text {Diff }}}=-1 \\
H_{k}^{\mathrm{C}_{\mathrm{n}}}=\frac{d \mathrm{OCV}}{d \mathrm{C}_{\mathrm{n}}}=\left.\frac{\partial \mathrm{OCV}}{\partial \mathrm{C}_{\mathrm{n}}}\right|_{\mathrm{C}_{\mathrm{n}}^{-}}+\frac{\partial \mathrm{OCV}}{\partial \mathrm{SOC}_{k}^{-}} \frac{d \mathrm{SOC}_{k}^{-}}{d \mathrm{C}_{\mathrm{n}}} \\
H_{k}^{\mathrm{R}_{\mathrm{i}}}=\frac{\partial V_{k}}{\partial \mathrm{R}_{\mathrm{i}}}=-i_{k}
\end{gathered}
$$

\section{PROPOSED APPROACH}

\section{A. Discharging/charging Voltage-temperature (DCVT) Pattern}

After fully charging (SOC 100\%) at a constant current of $4 \mathrm{~A}$, followed by a rest period, each cell was discharged to SOC $80 \%$. Using a scaled-down discharging/charging current profile of a HEV, shown in Fig. 4, ten DCVT patterns were collected. The DCVT pattern is recorded for ten fresh Li-Ion cells at various temperatures: $10-50^{\circ} \mathrm{C}$ with a $5^{\circ} \mathrm{C}$ temperature interval. For pattern recognition, each average DCVT for ten fresh Li-Ion cells at various temperatures are obtained and shown in Fig. 5. 

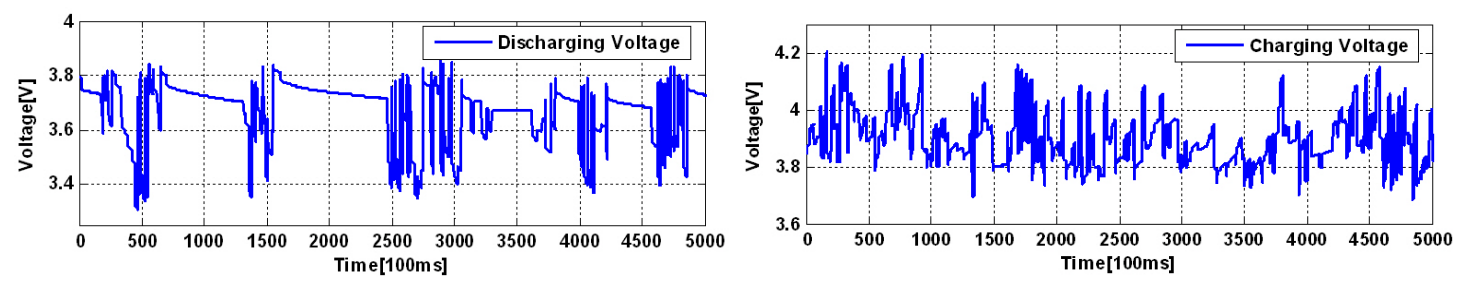

Fig. 6. Two patterns separated from the DCVT pattern. (a) Discharging-voltage temperature (DVT) pattern. (b) Charging-voltage temperature (CVT) pattern.
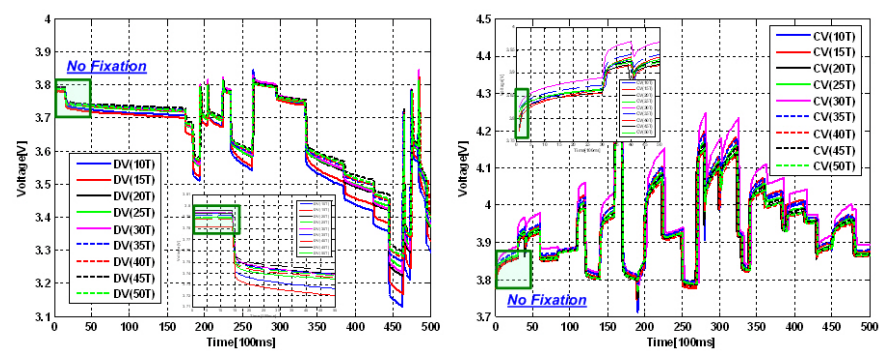

Fig. 7. Unfixed average discharging and charging voltages.

\section{B. Initial Starting Voltage Points (ISVP) Fixation}

For recognition of the DCVT pattern with the Hamming network, statistical analysis is necessary. First, all DCVT patterns are separated into two patterns according to current $i$ : discharging voltage-temperature (DVT; $i \geq 0)$ patterns and charging voltage-temperature (CVT; $i<0)$ patterns, as shown in Fig. 6. Second, the initial starting points of each DVT and CVT should be fixed. As shown in Fig. 7, the initial starting voltage points (ISVP) of the nine representative DCVT patterns are not fixed due to their various electrochemical characteristics at different temperatures. Hence, the averages and standard deviations of the collected discharging/charging voltages cannot be compared, and it is required to set a standard ISVP, as shown in Fig. 8. For example, consider three cells (A-C) with different ISVPs (VA1I-VC1I). It is required to choose one as the standard cell, B (VB1I=VB2I), and the other two voltages of $\mathrm{A}$ and $\mathrm{C}$ are up and down, respectively (VA1IVA2I, VC1IVC2I). Therefore, three ISVPs are fixed at one point. Based on this rule, the average and standard deviation for the voltage of A can be expressed in (22) and (23), respectively.

$$
\begin{gathered}
\text { Battery } \mathrm{AVE}_{\mathrm{A}}^{\mathrm{AVE}}=\frac{\sum_{\mathrm{i}=1}^{n} \mathrm{~V}_{\mathrm{A} 2 \mathrm{i}}}{n} \quad(n=1,2, \cdots, F) \\
\text { Battery }_{\mathrm{A}}^{\mathrm{STD}}=\sqrt{\frac{\sum_{\mathrm{i}=1}^{n}\left(\mathrm{~V}_{\mathrm{A} 2 \mathrm{i}}-\text { Battery }_{\mathrm{A}}^{\mathrm{AVE}}\right)^{2}}{n}} \quad(n=1,2, \cdots, F)
\end{gathered}
$$

In addition, for a given discharging condition, the average (AVE) and standard deviation (STD) of the three cells can be compared in (24) and (25), respectively.

$$
\begin{aligned}
& \text { Battery }_{\mathrm{A}}^{\mathrm{AVE}}>\text { Battery }_{\mathrm{B}}^{\mathrm{AVE}}>\text { Battery }_{\mathrm{C}}^{\mathrm{AVE}} \\
& \text { Battery }_{\mathrm{A}}^{\mathrm{STD}}<\text { Battery }_{\mathrm{B}}^{\mathrm{STD}}<\text { Battery }_{C}^{\mathrm{STD}}
\end{aligned}
$$

The fixed discharging/charging voltages are given in Fig. 9. All averages and standard deviations for the collected discharging/charging voltages can be compared through statistical analysis.
TABLE I

Seven Characteristics Parameters

\begin{tabular}{|c|c|l|}
\hline DCVT pattern & C1 & Standard deviation DCVT \\
\hline DVT pattern & C2 & Standard deviation DVT \\
\hline CVT pattern & C3 & Standard deviation CVT \\
\hline \multirow{2}{*}{ DVT pattern } & C4 & Average DVT (f) \\
\cline { 2 - 3 } & C5 & Standard deviation DVT (f) \\
\hline \multirow{2}{*}{ CVT pattern } & C6 & Average CVT (f) \\
\cline { 2 - 3 } & C7 & Standard deviation CVT (f) \\
\hline \multicolumn{2}{|c|}{ Standard $: 25^{\circ} \mathrm{C}$ (discharging/charging) (f) : fixation } \\
\hline
\end{tabular}

\section{Characteristic Parameters of the DCVT Pattern}

As indicated in Table I, characteristic parameters C1 to C7 are learned by the Hamming network using the average and standard deviations based on DCVT, DVT, and CVT patterns.

Each value of the seven characteristic parameters corresponding to the nine representative DCVT patterns is transformed into 1 and -1 element array with four levels, as shown in Fig. 10. If these patterns are not transformed into this binary form, then the pattern recognition performance can be distorted by a large real-valued parameter. In Fig. 10, avg is the average and std is the standard deviation of each characteristic parameter. The levels of each parameters are decided by three standard, viz., $a v g-(\alpha \times s t d), m$, and $a v g+(\alpha \times s t d)$. The levels are decided according to the parameter values, as shown in Fig. 11. For example, if the value is larger than avg- $(\alpha \times s t d)$ and smaller than avg, the level is L3, and if the value is larger than avg and smaller than $a v g+(\alpha \times s t d)$, the level is L2. $\alpha$ is a tuning value and is chosen as 0.5 to make the characteristic difference of nine representative patterns.

\section{Pattern Recognition with the Hamming Network}

As shown in Fig. 12, the feedforward layer calculates the inner product between each representative pattern and the current pattern. The seven characteristic parameters corresponding to nine representative DCVT patterns are transformed into the binary form and stored in the weight matrix $\mathbf{W}^{1}$. The nine neurons storing the results of the inner product in the feedforward layer compete with each other to determine a winner. After this process, only one neuron will have a nonzero output, and this neuron indicates the representative pattern closest to the current pattern.

\section{E. Temperature-checking Process}

The representative DCVT pattern closest to an arbitrary DCVT pattern is selected. The outputs for the two layers of the Hamming network for three unknown DCVT patterns are shown in Figs. 13, 17, and 18. As shown in Fig. 13(b), the selected DCVT pattern closest to an arbitrary DCVT pattern 


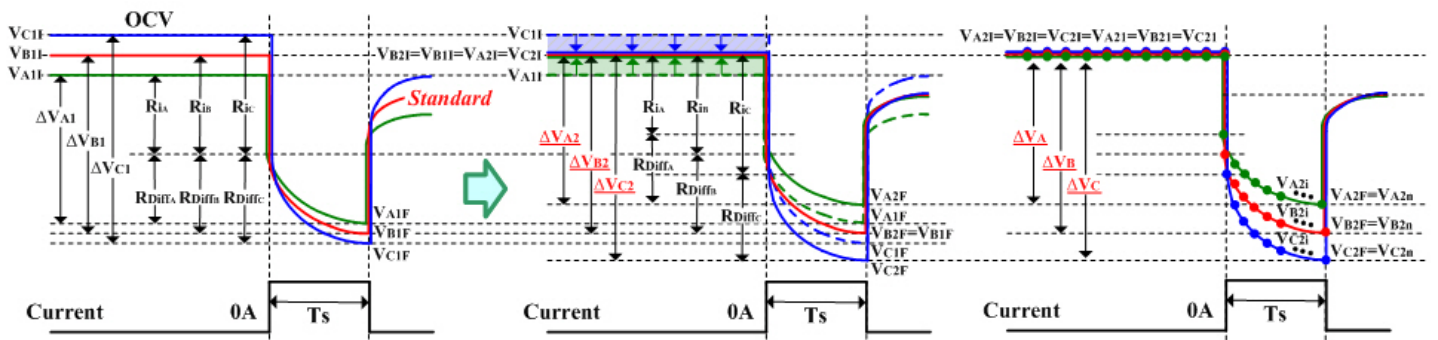

Fig. 8. Initial starting voltage points (ISVP) fixation.
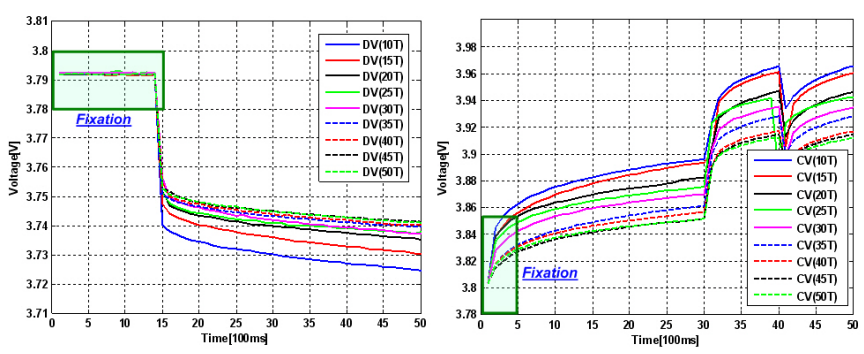

Fig. 9. Fixed average discharging and charging voltages.

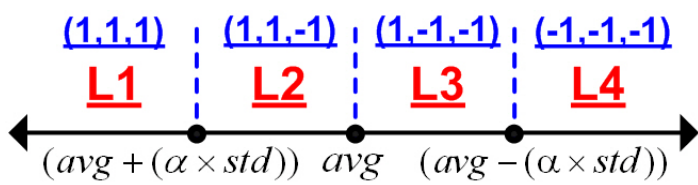

Fig. 10. Four levels as to three standards.

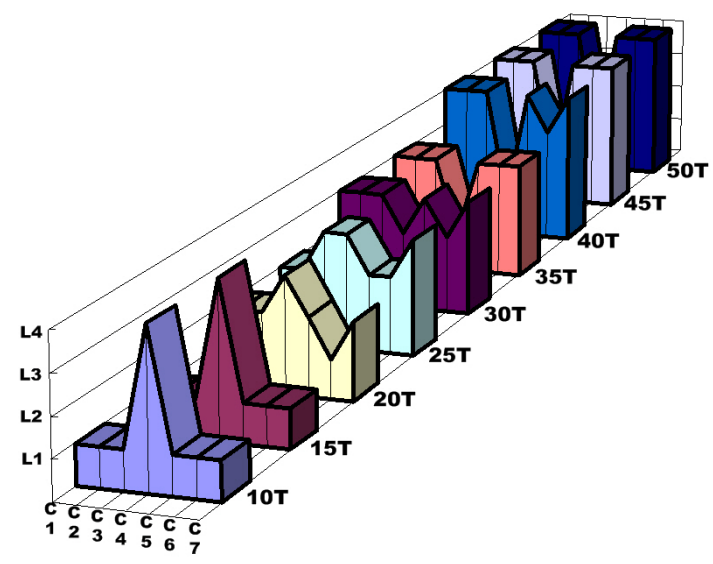

Fig. 11. Characteristics of nine representative patterns.

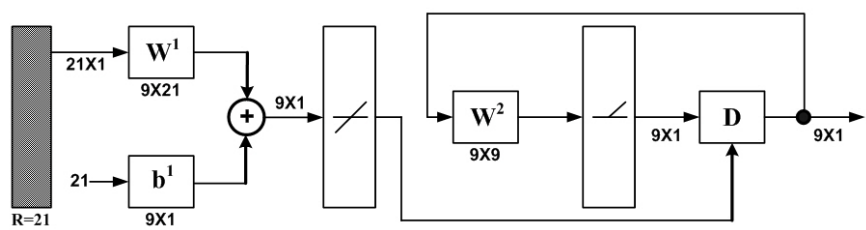

Fig. 12. Hamming network in this study.

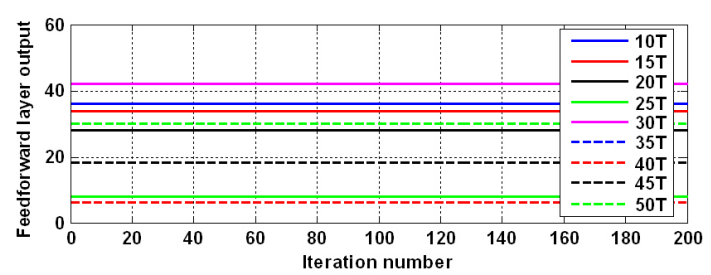

(a)

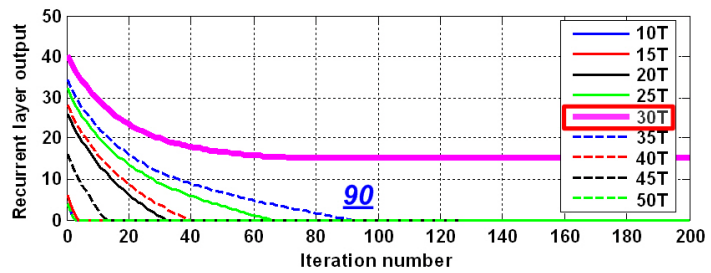

(b)

Fig. 13. Outputs of two neural network layers (arbitrary 1, selected pattern $30^{\circ} \mathrm{C}$ ). (a) Feedforward layer. (b) Recurrent layer.

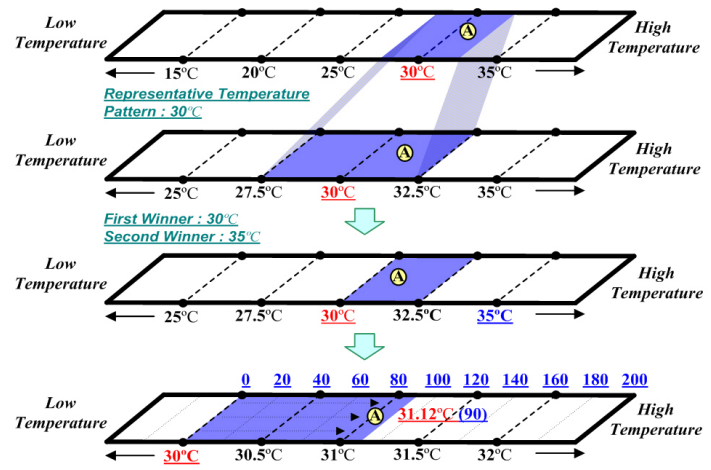

Fig. 14. Temperature-checking process.

1 is the DCVT pattern of $30^{\circ} \mathrm{C}$ as the closest match. The second- closest match is the DCVT pattern of $35^{\circ} \mathrm{C}$. As aforementioned, one neuron that has a non-zero output is only selected as the first winner, or best fit, or the winnertake-all (WTA) operation. Then, two neurons with similar characteristics compete before the end of the selection of the first winner. Large differences in characteristics between two neurons lead to fast convergence to zero for the second winner. Conversely, small characteristic differences in characteristics between two neurons lead to slow convergence to zero. Based on this rule of the Hamming network, it is possible to check the approximate experimental temperature without a temperature sensor. The procedure of the temperature-checking process based on the Hamming network is shown in Fig. 14. When an 


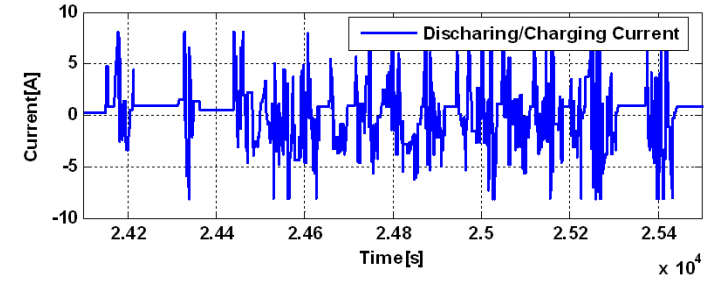

(a)

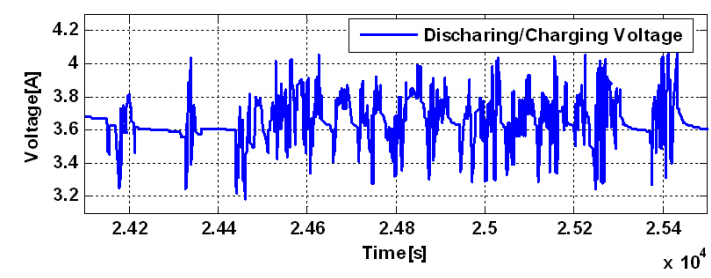

(b)

Fig. 15. Two plots for discharging/charging voltage-temperature (DCVT) pattern at an unknown temperature. (a) Current profile. (b) Voltage data.

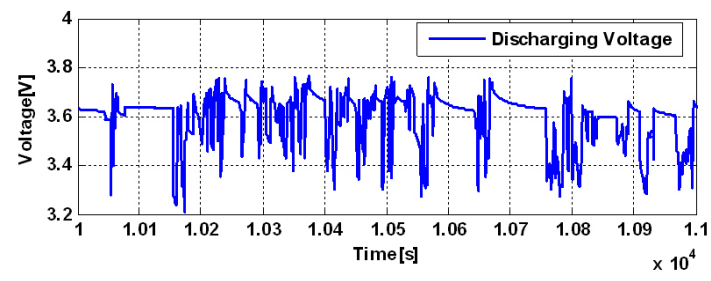

(a)

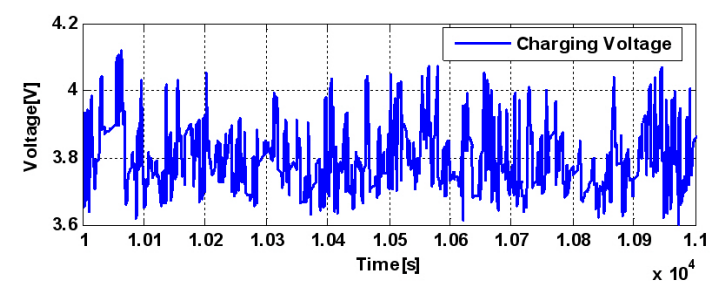

(b)

Fig. 16. Two separated voltages from the DCVT pattern at an unknown temperature. (a) Discharging voltage-temperature (DVT) pattern. (b) Charging voltage-temperature (CVT) pattern.

arbitrary DCVT patterns is applied as the input pattern, one DCVT pattern of $30^{\circ} \mathrm{C}$ is selected as the closest representative to the arbitrary DCVT pattern. Due to the $5^{\circ} \mathrm{C}$ temperature interval under experimental conditions, it is well known that the experimental temperature is in the range of $27.5^{\circ} \mathrm{C}$ to $32.5^{\circ} \mathrm{C}$. In addition, as shown in Fig. 13(b), the other DCVT pattern of $35^{\circ} \mathrm{C}$ for the second winner is determined according to the competition result. Therefore, the experimental temperature can be expected in the range of $30.5^{\circ} \mathrm{C}$ to $32.5^{\circ} \mathrm{C}$. For reference, if the other DCVT pattern of $25^{\circ} \mathrm{C}$ was the second winner, the expected range would be $27.5^{\circ} \mathrm{C}$ to $30.0^{\circ} \mathrm{C}$. To accurately check the experimental temperature, the number of iterations when the recurrent layer output for the second winner is zero, after the competition, is required. As shown in Fig. 13(b), intercept of the recurrent layer output on the iteration-axis (0-200) for the second winner indicates the number of iterations. According to the expected temperature range of $2.5^{\circ} \mathrm{C}$, the difference in elevated or reduced temperatures per one unit on the iteration-axis is $0.0125^{\circ} \mathrm{C}\left(2.5^{\circ} \mathrm{C} / 200\right)$.

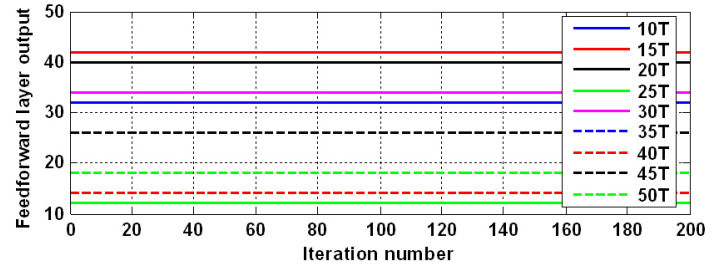

(a)

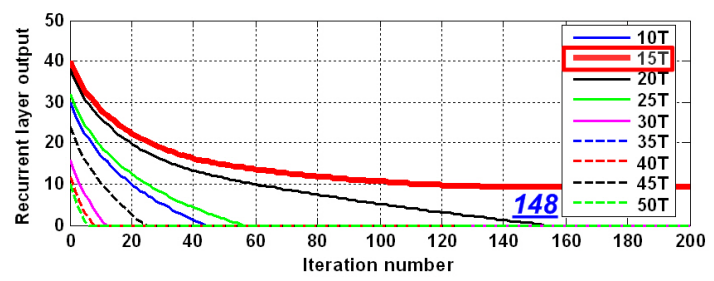

(b)

Fig. 17. Outputs of two neural network layers (arbitrary 2, selected pattern $15^{\circ} \mathrm{C}$ ). (a) Feedforward layer. (b) Recurrent layer.

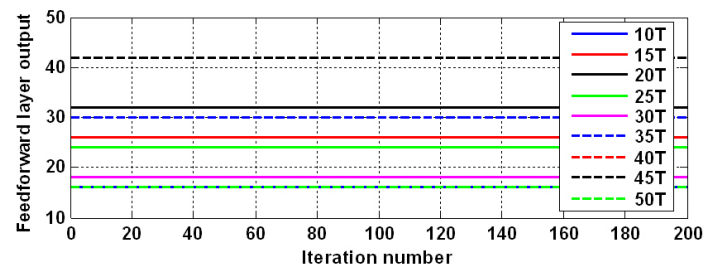

(a)

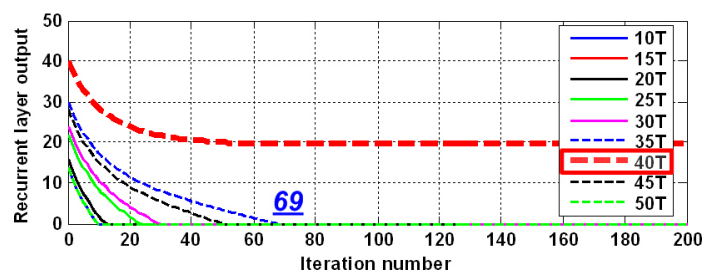

(b)

Fig. 18. Outputs of two neural network layers (arbitrary 3, selected pattern $40^{\circ} \mathrm{C}$ ). (a) Feedforward layer. (b) Recurrent layer.

The starting-temperature point can be determined based on the selected DCVT pattern for the first winner. With the number of iterations, the difference in elevated or reduced temperatures can be obtained. In Fig. 13, the number of iterations to convergence to zero on the iteration-axis is 90 . Given the first winner of the DCVT $\left(30^{\circ} \mathrm{C}\right)$, the elevated temperature is $1.12^{\circ} \mathrm{C}(0.012590)$. Therefore, the estimated temperature result using the temperature-checking process for SOC/capacity estimation and $\mathrm{SOH}$ prediction using the DEKF is $31.12^{\circ} \mathrm{C}$. This shows little difference when compared to the real temperature of $31^{\circ} \mathrm{C}$. The measured empirical parameters measured in advance are modified and used as model parameters [15].

\section{VERIFICATION}

\section{A. SOC/Capacity Estimation}

In this section, SOC/capacity estimation results based on pattern recognition combined with empirical parameters are shown in detail. To verify the proposed approach, SOC/ 
TABLE II

Numbers of ITERATION, TEMPERATURE EsTIMATED AND DiRECTLY MEASURED

\begin{tabular}{|c|c|c|c|}
\hline Number of iteration & 90 & 148 & 69 \\
\hline First/Second winners & $30^{\circ} \mathrm{C} / 25^{\circ} \mathrm{C}$ & $15^{\circ} \mathrm{C} / 20^{\circ} \mathrm{C}$ & $40^{\circ} \mathrm{C} / 35^{\circ} \mathrm{C}$ \\
\hline Temperature estimated $\left[{ }^{\circ} \mathrm{C}\right]$ & $31.12^{\circ} \mathrm{C}$ & $16.85^{\circ} \mathrm{C}$ & $39.14^{\circ} \mathrm{C}$ \\
\hline Temperature measured $\left[{ }^{\circ} \mathrm{C}\right]$ & $31^{\circ} \mathrm{C}$ & $17^{\circ} \mathrm{C}$ & $39^{\circ} \mathrm{C}$ \\
\hline
\end{tabular}

TABLE III

RESULTS OF ESTIMATED CAPACITY IN DEKF AND EXPERIMENTAL Capacities Directly Measured

\begin{tabular}{|c|c|c|c|}
\hline Cell & $\begin{array}{c}\text { Arbitrary 1 } \\
\left(31.12^{\circ} \mathrm{C}\right)\end{array}$ & $\begin{array}{c}\text { Arbitrary 2 } \\
\left(16.85^{\circ} \mathrm{C}\right)\end{array}$ & $\begin{array}{c}\text { Arbitrary 3 } \\
\left(39.14^{\circ} \mathrm{C}\right)\end{array}$ \\
\hline Capacity estimated $[\mathrm{Ah}]$ & 1.3184 & 1.1733 & 1.2874 \\
\hline Capacity measured $[\mathrm{Ah}]$ & 1.3173 & 1.1751 & 1.2861 \\
\hline
\end{tabular}

capacity estimation are performed at three experimental temperatures determined by the temperature-checking process.

Fig. 15(a) shows another scaled-down HEV automotive current profile was applied to the cell. The collected DCVT pattern shown in Fig. 15(b) was separated into the discharging and charging voltages, as shown in Fig. 16. Next, for the three unknown temperatures, based on the Hamming network, including the temperature-checking process, the suitable experimental temperature is determined. According to these temperatures, the pattern changes due to model parameter variation are implemented at an unknown temperature and compared with those at room temperature of $25^{\circ} \mathrm{C}$, and used to implement a few simple techniques [15]. Finally, the obtained model parameters are used for SOC/capacity estimation. Three determined temperature results based on temperature-checking process are shown in Figs. 13(b), 17(b), and 18(b). For the results, the number of iterations for convergence to zero on the iteration-axis is 90,148 , and 69 , respectively.

The number of iterations and determined temperature using the first winner pattern are shown in Table II. The estimated temperature is determined from the first winners of each recurrent layer and the number of iterations. The SOC and capacity estimate based on the DEKF for three unknown cells are compared with those of ampere-hour counting and measured capacity. Measurements are shown in Fig. 19, and results are summarized in Table III. At any temperature, the SOC and capacity estimation results of the DEKF satisfy the specification within $\pm 5 \%$.

\section{B. SOH Prediction}

The direct current internal resistance (DCIR) [16] is defined as the magnitude of the lumped parameter, namely $\mathrm{R}_{i}+\mathrm{R}_{\text {Diff }}$, is used to obtain the $\mathrm{SOH}$ based on the pulse power analysis [7], [8]. Therefore, in order to overcome erroneous $\mathrm{SOH}$ prediction, it is important to know the DCIR variance at various temperatures. The DCIR experiments were carried out for DCIR measurement at various temperatures using 10 fresh Li-Ion cells previously used to obtain the DCVT pattern. The average constant DCIRs of 10 fresh Li-Ion cells at SOC 60\% are shown in Fig. 20. The DCIR increased with a decrease of temperature. Specifically, the marked difference in DCIR among the cells is shown below room temperature and more increased at reduced temperatures of $10-20^{\circ} \mathrm{C}$. The pattern changes of the DCIR at a specified temperature when compared with that at room temperature are used to implement
TABLE IV

Maxd_Coef, Mind_Coef, AND Temp_Coef VALUeS AT VARIOUS TEMPERATURES

\begin{tabular}{|c|c|c|c|c|c|}
\hline Temperatures & $10^{\circ} \mathrm{C}$ & $15^{\circ} \mathrm{C}$ & $20^{\circ} \mathrm{C}$ & $25^{\circ} \mathrm{C}$ & $30^{\circ} \mathrm{C}$ \\
\hline Maxd_Coef & 0.9086 & 0.9096 & 0.9105 & 0.9357 & 0.9520 \\
\hline Mind_Coef & 1.0713 & 1.0654 & 1.0595 & 1.0554 & 1.0307 \\
\hline Temp_Coef & 0.10 & 0.15 & 0.15 & 0.20 & 0.15 \\
\hline \hline Temperatures & $35^{\circ} \mathrm{C}$ & $40^{\circ} \mathrm{C}$ & $45^{\circ} \mathrm{C}$ & $50^{\circ} \mathrm{C}$ & \\
\hline Maxd_Coef & 0.9662 & 0.9805 & 0.9813 & 0.9822 & \\
\hline Mind_Coef & 1.0291 & 1.0275 & 1.0241 & 1.0208 & \\
\hline Temp_Coef & 0.10 & 0.05 & 0.05 & 0.05 & \\
\hline
\end{tabular}

TABLE V

DCIRs of Fully Fresh AND AGED CELlS AT VARIOUS TEMPERATURES

\begin{tabular}{|c|c|c|}
\hline Temperature & Fully fresh cell DCIR $[\Omega]$ & Fully aged cell DCIR $[\Omega]$ \\
\hline $10^{\circ} \mathrm{C}$ & 0.12896 & 0.19724 \\
\hline $20^{\circ} \mathrm{C}$ & 0.07921 & 0.12617 \\
\hline $25^{\circ} \mathrm{C}$ & 0.05896 & 0.09875 \\
\hline $30^{\circ} \mathrm{C}$ & 0.05586 & 0.08719 \\
\hline $40^{\circ} \mathrm{C}$ & 0.04229 & 0.06824 \\
\hline $50^{\circ} \mathrm{C}$ & 0.03832 & 0.06221 \\
\hline
\end{tabular}

the DCIR-temperature relation for SOH prediction. First of all, given average, maximum, and minimum DCIRs among $10 \mathrm{Li}-$ Ion cells at various temperatures, Maxd_Coef and Mind_Coef can be expressed in Eqs. (26) and (27), respectively.

$$
\begin{aligned}
& \text { Maxd_Coef }=\frac{\text { Average DCIR }}{\text { Maximum DCIR }}<1 \\
& \text { Mind_Coef }=\frac{\text { Average DCIR }}{\text { Minimum DCIR }}>1
\end{aligned}
$$

Nine coefficients corresponding to experimental temperature range are each determined at various experimental temperatures $\left(10-50^{\circ} \mathrm{C}\right.$ with a $5^{\circ} \mathrm{C}$ temperature interval), shown in Fig. 21 and Table IV. Considering a weighted coefficient Temp_Coef at experimental temperature range of $10-50^{\circ} \mathrm{C}$, the Maxd(mod) and Mind(mod) can be computed from the sum of the multiplication between Maxd_Coef(Mind_Coef) and Temp_Coef in Eqs. (28) and (29), respectively.

$$
\begin{aligned}
& \text { Maxd }(\mathrm{mod})=\text { Maxd_Coef } \times \text { Temp_Coef }=1.0481 \\
& \quad\left(10 \sim 50^{\circ} \mathrm{C} ; 5^{\circ} \mathrm{C} \text { interval }\right) \\
& \text { Mind }(\bmod )=\text { Mind_Coef } \times \text { Temp_Coef }=0.9376 \\
& \left(10 \sim 50^{\circ} \mathrm{C} ; 5^{\circ} \mathrm{C} \text { interval }\right)
\end{aligned}
$$

As expressed in (30), the $\mathrm{SOH}$ of an arbitrary cell can be predicted using the selected cell pattern's DCIR, DCIR selected .

$$
\underset{\text { arbitrary }}{S O H}=\left|\frac{D C I R_{\text {selected }}-D C I R_{\text {aged }}}{D C I R_{\text {fresh }}-D C I R_{\text {aged }}}\right|
$$

where $\mathrm{DCIR}_{\text {fresh }}$ and DCIR ${ }_{\text {aged }}$ are each DCIRs of fully fresh and aged cells among the 1.3Ah group. Under the identical experimental condition of Fig. 20, each DCIR of fully fresh and aged cells are listed in Table V.

Using the determined temperature $\left(31.12^{\circ} \mathrm{C}\right)$ based on the temperature-checking process, the $\mathrm{SOH}$ of an arbitrary cell can be obtained. The procedure is shown in Table VI.

\section{CONCLUSIONS}

Precise SOC/capacity estimation and $\mathrm{SOH}$ prediction are critical for practical applications and to prevent over-charging 


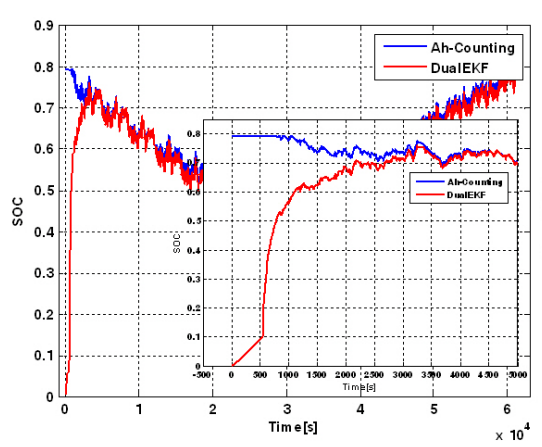

(a)

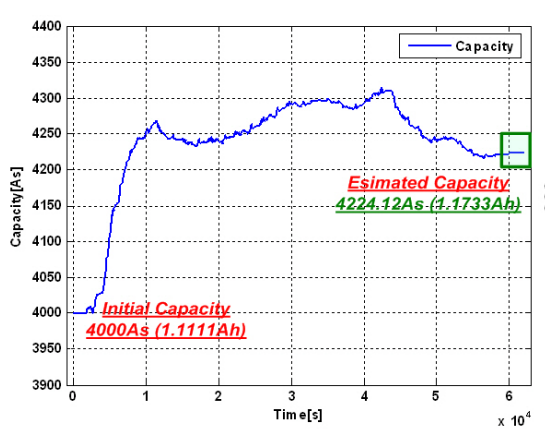

(d)

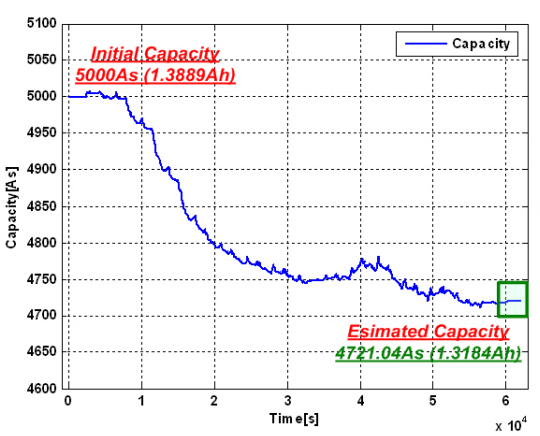

(b)

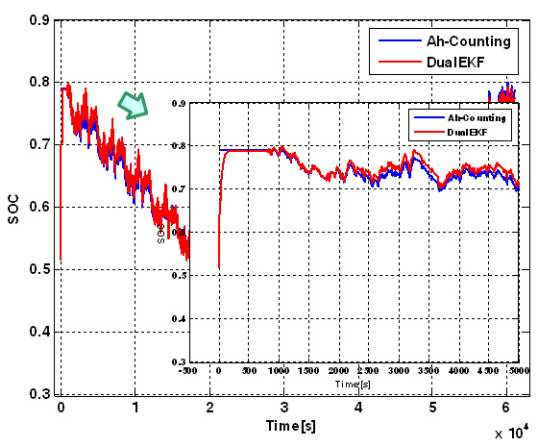

(e)

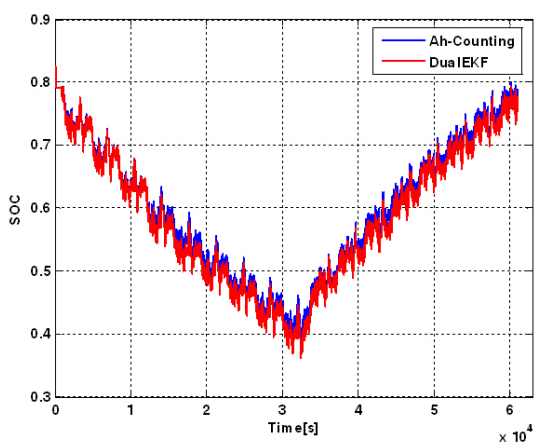

(c)

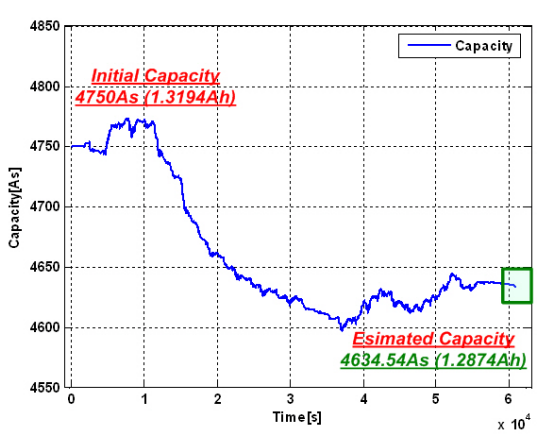

(f)

Fig. 19. SOC/capacity estimation based on DEKF in comparison with ampere-hour counting and measured capacity. (a)(b) SOC (Initial SOC 0), Capacity (Initial capacity $1.3889 \mathrm{Ah}$ ); Unknown cell 1 , Arbitrary temperature $31^{\circ} \mathrm{C}$, selected pattern $30^{\circ} \mathrm{C}$. (c)(d) SOC (Initial SOC 0.793 ), Capacity (Initial capacity $1.1111 \mathrm{Ah}$ ); Unknown cell 2, Arbitrary temperature $17^{\circ} \mathrm{C}$, selected pattern $15^{\circ} \mathrm{C}$. (e)(f) SOC (Initial SOC 0.5), Capacity (Initial capacity $1.3194 \mathrm{Ah}$ ); Unknown cell 3, Arbitrary temperature $39^{\circ} \mathrm{C}$, selected pattern $40^{\circ} \mathrm{C}$.

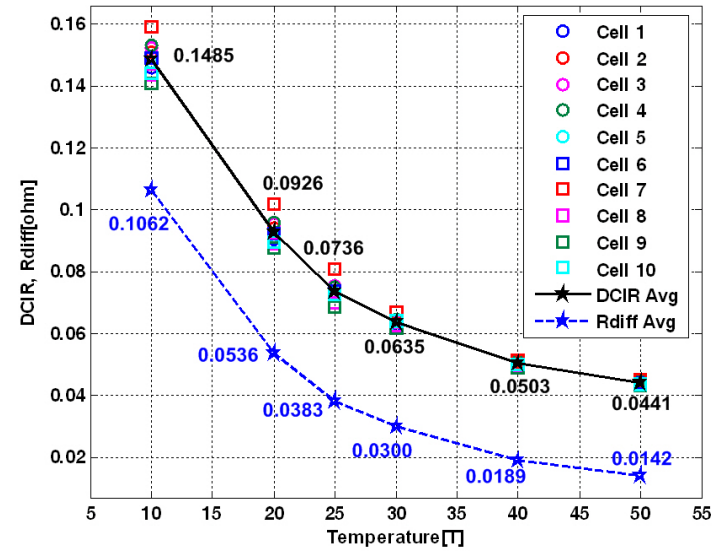

Fig. 20. Average values of the DCIR at SOC $60 \%$ (temperatures: $10-50^{\circ} \mathrm{C}$ with a $10^{\circ} \mathrm{C}$ temperature interval.
TABLE VI

PROCEDURE FOR OBTAINING THE SOH OF AN UNKNOWN CELL 1 AFTER THE TEMPERATURE-CHECKING PROCESS $\left(31.12^{\circ} \mathrm{C}\right.$, SOC $\left.27 \%\right)$

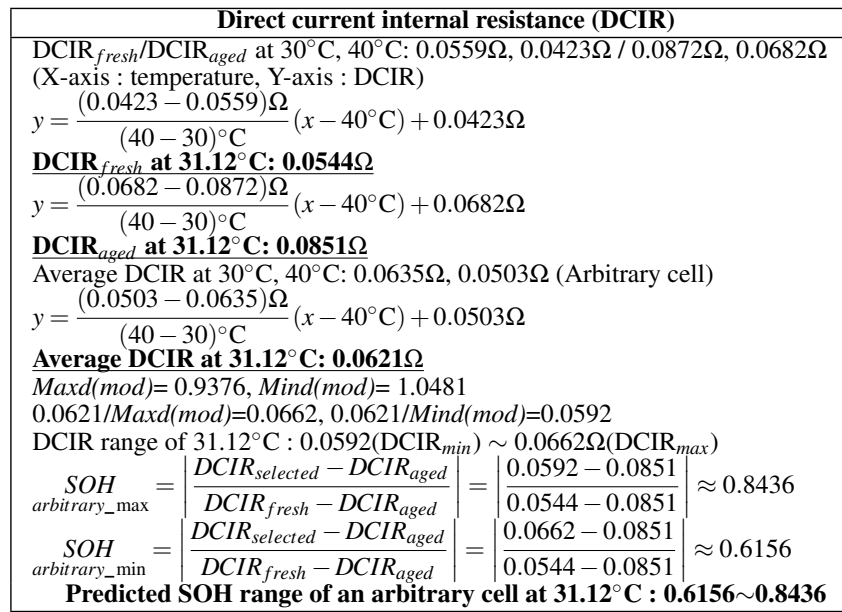

and over-discharging, which may cause permanent internal damage. This study presents an application of the Hamming network-DEKF based on pattern recognition for improved $\mathrm{SOC} /$ capacity estimation and $\mathrm{SOH}$ prediction at various temperatures. This method avoids the need for repeated measurement of cell parameters before SOC/capacity estimation and $\mathrm{SOH}$ prediction process. 


\section{ACKNOWLEDGMENT}

This work was supported by the New and Renewable Energy Program of the Korea Institute of Energy Technology Evaluation and Planning (KETEP) grant funded by the Korea government Ministry of Knowledge Economy (NO. 20104010100490).

\section{REFERENCES}

[1] H.-S. Park, C.-E. Kim, C.-H. Kim, G.-W. Moon, and J.-H. Lee, "A Modularized Charge Equalizer for an HEV Lithium-Ion Battery String," IEEE Trans. Ind. Electron., Vol. 56, No. 5, pp. 1464-1476, May. 2009.

[2] G. L. Plett, "Recursive approximate weighted total least squares estimation of battery cell total capacity," J. Power Sources, Vol. 196, No. 4, pp. 2319-2331, Feb. 2011.

[3] J. Wang, B. Cao, Q. Chen, and F. Wang, "Combined state of charge estimator for electric vehicle battery pack," Control Eng. Pract., Vol. 15, No. 12, pp. 3182-3186, Dec. 2007.

[4] N. A. Monfared, N. Gharib, H. Moqtaderi, M. Hejabi, M. Amiri, F. Torabi, and A. Mosahebi, "Prediction of state-of-charge effects on leadacid battery characteristics using neural network parameter modifier," $J$. Power Sources, Vol. 158, No. 2, pp. 932-935, Aug. 2006.

[5] W. H. Shen, "State of available capacity estimation for lead-acid batteries in electric vehicles using neural network," Energy Conv. Manag., Vol 48, No. 2, pp. 433-442, Feb. 2007.

[6] K. T. Chau, K.C. Wu, and C. C. Chan, "A new battery capacity indicator for lithium-ion battery powered electric vehicles using adaptive neurofuzzy inference system," Energy Conv. Manag., Vol. 45, No. 11-12, pp. 1681-1692, Jul. 2004.

[7] D. P. Abraham, J. Liu, C. H. Chen, Y. E. Hyung, M. Stoll, N. Elsen, S. MacLaren, R. Twesten, R. Haasch, E. Sammann, I. Petrov, K. Amine, and G. Henriksen, "Diagnosis of power fade mechanisms in high-power lithium-ion cells," J. Power Sources, Vol. 119-121, No. 1, pp. 241-246, Jun. 2003.

[8] E. V. Thomas, H. L. Case, D. H. Doughty, R. G. Jungst, G. Nagasubramanian, and E. P. Roth, "Accelerated power degradation of Li-ion cells," J. Power Sources, Vol. 124, No. 1, pp. 254-260, Oct. 2003.

[9] V. Agarwal, K. Uthaichana, R. A. Decarlo, L. H. Tsoukalas, "Development and validation of a battery model useful for discharging and charging power control and lifetime estimation," IEEE Trans. Energy. Convers., Vol. 25, No. 3, pp. 821-835, Sep. 2010.

[10] G. L. Plett, "Extended Kalman filtering for battery management systems of LiPB-based HEV battery packs : Part 1-3," J. Power Sources, Vol. 134, No. 2, pp. 252-292, Aug. 2004.

[11] S.-J. Lee, J.-H. Kim, J.-M. Lee, and B.H. Cho, "State-of-charge and capacity estimation of lithium-ion battery using a new open-circuit voltage versus state-of-charge," J. Power Sources, Vol. 185, No. 2, pp. 1367-1373, Dec. 2008.

[12] J.-M. Lee, O.-Y. Nam, and B.H. Cho, "Li-ion battery SOC estimation method based on the reduced order extended Kalman filtering," J. Power Sources, Vol. 174, No. 1, pp. 9-15, Nov. 2007.

[13] K. Koutroumbas, and N. Kalouptsidis, "Generalized Hamming networks and applications," Neural Netw., Vol. 18, No. 7, pp. 896-913, Sep. 2005.
[14] M. T. Hagan, H. B. Demuth, and M. B, Neural network Design. Boston StateMA: PWS Publishing Co. 1995.

[15] J.-H. Kim, S.-J. Lee, and B.H. Cho, "The State of Charge Estimation Employing Empirical Parameters Measurement for Various Temperature," in Proc. of IEEE Int. Power Electron. Motion Control Conf., pp. 393-944, May 2009.

[16] J.-H. Kim, S.-J. Lee, J.-M. Lee, and B. H. Cho, "A New Direct Current Internal Resistance and State of Charge Relationship for the Li-Ion Battery Pulse Power Estimation," in Proc. of IEEE Int. Conf. Power Electron., pp. 1173-1178, Oct. 2007.

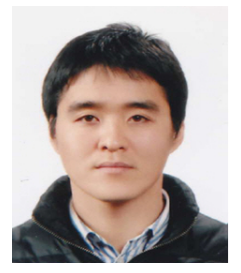

Jonghoon Kim received the B.S. in Electrical Engineering from Chungnam National University (CNU), Daejeon, Republic of Korea, in 2005 and is currently pursuing both his M.S. and Ph.D. at Seoul National University (SNU), Seoul, Republic of Korea. He received the Student Paper Award from IPEC-10. His main research interests include battery management system (BMS; modeling, screening, equalization, and $\mathrm{SOC} / \mathrm{SOH}$ estimation), and fuel cell system (ripple current analysis, $\mathrm{SOH}$ prediction, modeling). He is a graduate student member of the IEEE and the member of the Korean Institute of Power Electronics (KIPE).

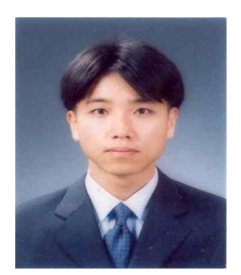

Seongjun Lee received the B.S. degree in electrical engineering from Kwangwoon University, Seoul, Korea, in 2004 and the M.S. and Ph.D. degrees from Seoul National University (SNU), Seoul, Republic of Korea, in 2006 and 2011, respectively. He is currently working as a senior research engineer in Defence Program Division, SAMSUNG TECHWIN. His main research interests include modeling, analysis and control of power electronics systems for hybrid electric vehicle (HEV) and electric vehicle(EV), battery management system (BMS), and distributed power system. He is a member of the IEEE and the member of the Korean Institute of Power Electronics (KIPE).

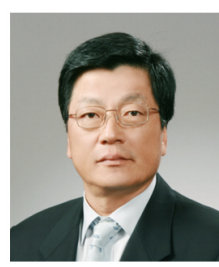

Bohyung Cho received the B.S. and M.S. degrees from California Institute of Technology, Pasadena, and the $\mathrm{Ph} . \mathrm{D}$. degree from Virginia Polytechnic Institute and State University (Virginia Tech), Blacksburg, all in electrical Engineering. Prior to his research at Virginia Tech, he worked as a member of Technical Staff with the Power Conversion Electronics Department, TRW Defense and Space System Group. From 1982 to 1995, he was a Professor with the Department of Electrical Engineering, Virginia Tech. In 1995, he joined School of Electrical Engineering, Seoul National University, Seoul, Korea, where he is currently a Professor His current research interests include power electronics, modeling, analysis, and control of spacecraft power processing equipment, and distributed power systems. Dr. Cho was a recipient of the 1989 Presidential Young Investigator Award from the National Science Foundation. He chaired the 2006 IEEE Power Electronics Specialists Conference (PESC 2006). He is a member of Tau Beta Pi. 